\title{
Use of Unmanned Aerial Vehicle to Execute Water Conservation Design for Hillsides
}

\author{
Wei-Ling Hsu, ${ }^{1}$ Ji-Yun Jhuang, ${ }^{2}$ Yi-Sian Chen, ${ }^{3}$ \\ Yan-Chyuan Shiau, ${ }^{4 *}$ and Tian-Yow Chern ${ }^{5}$ \\ ${ }^{1}$ Huaian Key Laboratory of Geographic Information Technology and Applications, \\ School of Urban and Environmental Science, Huaiyin Normal University, \\ No. 111, ChangJang West Road, Huai'an City, Jiangsu Province 223300, China \\ ${ }^{2} \mathrm{Ph}$. D. Program of Technology Management, Chung Hua University, \\ No. 707, WuFu Road, Section 2, Hsinchu 30012, Taiwan \\ ${ }^{3}$ Department of Civil Engineering, Chung Hua University, No. 707, WuFu Road, Section 2, Hsinchu 30012, Taiwan \\ ${ }^{4}$ Department of Landscape Architecture, Chung Hua University, \\ No. 707, WuFu Road, Section 2, Hsinchu 30012, Taiwan \\ ${ }^{5}$ Department of Architecture and Urban Planning, Chung Hua University, \\ No. 707, WuFu Road, Section 2, Hsinchu 30012, Taiwan \\ (Received October 30, 2018; accepted December 25, 2018)
}

Keywords: water and soil conservation, UAV, 3D models, hillsides, digital camera

The applications of remote sensing technology and aerial photography using unmanned aerial vehicles (UAVs) have facilitated the development of large-scale land environmentmonitoring technology. When design units are involved in water and soil conservation projects, engineering personnel must take valuable instruments to valleys and streams for mapping purposes; however, obtaining effective and accurate worksite information is challenging. In this study, a remote-controlled helicopter equipped with a digital camera was used to take photographs of riverside slopes that are unreachable by land. Three-dimensional (3D) models of rivers and mountain slopes were constructed through software computation. The results of this study thus provide references for design projects and show how inaccessibility problems, caused by factors such as a difficult terrain, can be solved, thereby avoiding disasters that can occur when surveying dangerous areas. The method effectively saves labor and time to ensure that hillside-related projects can be completed on schedule.

\section{Introduction}

Hillside slopes are among the key factors closely related to soil and water losses. ${ }^{(1)}$ A remote sensing system can detect surface reflection spectra from which surface characteristics can be elucidated. In recent studies, spectra covering various wavelengths and obtained from satellite and unmanned aerial vehicle (UAV) images have been employed as monitoring parameters. Spectral data generated by on-site sampling have been compared with data indicators to confirm the accuracy of surface monitoring technologies. ${ }^{(2)}$ They are one of the most important pieces of information in many natural-source spatial databases. ${ }^{(3)}$ An engineering and technical

*Corresponding author: e-mail: ycshiau@ms22.hinet.net https://doi.org/10.18494/SAM.2019.2172 
consulting and service firm is involved in new construction, expansion, reconstruction, and restoration processes. Its technical service process can be divided into various stages such as the conduct of a feasibility study, planning, design, construction, maintenance management, and demolition and reconstruction. Each stage requires the investment of manpower from various fields and different levels of material resources in order to avoid excessive resource consumption and effectively enhance construction quality and utility. The application of the new remote sensing measurement technology can improve the quality of soil and water conservation services.

\subsection{Water and soil conservation technology services}

Recently, applications of satellite remote sensing technology and aerial photography using UAVs have facilitated the development of environment-monitoring technology. Such technologies enable us to efficiently gather large-scale monitoring data at different time points. Changes in land use and land cover are critical driving forces behind variations in atmosphere, climate, and the ecosystem. ${ }^{(4)}$ The main elements of a soil and water conservation project at the initial stage are a feasibility study and planning, whereby engineers select appropriate routes and work sites after interpreting and analyzing basic information such as satellite images and aerial photomaps. Each worksite is then visited for investigation and confirmation. The work at this stage is generally difficult and dangerous because worksites are situated in remote locations, forcing engineers to carry valuable instruments and possibly traverse mountains to reach their destination, after which they may face wasteland, deep valleys, or streams. Engineers cannot reach these locations without suitable engineering measures (such as temporary roads and bridges for construction), and only recording equipment with telephoto lenses can be employed to photograph worksites. Such image data can only be used for reference and have extremely low application value. Completing subsequent planning and design by relying only on such data causes problems such as improper design and miscalculated construction expenditure.

\subsection{UAVs}

Depending on the sensors and platforms used during the remote sensing test, the science of remote sensing technology can be as expensive as that of satellite and aerial remoting sensing, or as inexpensive as or less costly than human observation. ${ }^{(5,6)}$ Technological advancements have enabled a wider range of tasks that can be completed by UAVs, which have functions ranging from entertainment to capturing images, providing transport resources, and detecting geographical topography in inaccessible areas. Capturing images of a wide area yields results that cannot be obtained by the human eye. ${ }^{(7)}$ With the development of remote sensing technology, a new data capturing method can provide significant convenience for digital recording and display. ${ }^{(8)}$ Alternatively, the use of UAVs with optical or laser sensors has been shown to support forest inventories, but is limited in scale (100 to 1000 ha). ${ }^{(9,10)}$ A UAV is generally any type of remote-controlled aircraft that does not require an onboard pilot, and it commonly refers to unmanned military reconnaissance aircraft. UAVs are generally divided into two categories: 
(a) Fixed wing: similarly to gliders, they are energy saving (fuel/electricity), incapable of fixed point hovering, and must fly at a specific altitude to avoid blurry images. They are suitable for large-area two-dimensional (2D) mapping.

(b) Rotary wing: more energy consuming, capable of fixed point hovering in air as well as vertical takeoff and landing, capable of oblique photography, suitable for small-area measurements or the three-dimensional (3D) modeling of single objects.

\subsection{Research objectives}

To effectively assist the design units, 3D models of hillsides that closely resemble the actual site of a water and soil conservation project were constructed. In this study, we employed the DJI Phantom 2 Vision+ UAV, which was flown at a predetermined altitude above the target area and used to take orthoimages. After the flight, the images were imported into the Pix4Dmapper software for computation in order to obtain point cloud files of the target area and reconstruct a visualized digital surface model (DSM).

A 3D model of the hillsides can be rapidly constructed after obtaining photographs of hillsides using a UAV. This method can be used to eliminate the difficult travel needed to complete a survey. The proposed method will aid in on-site surveying to avoid potential accidents in dangerous areas. The UAV can increase the surveying accuracy and reduce the design imperfections due to inadequate information. The proposed method can effectively save manpower and time so that hillside design can be completed on schedule.

The remainder of this paper is organized as follows. In Sect. 2, measurement classifications are shown in detail; in Sect. 3, an outline of the domestic applications of UAVs is given; in Sect. 4 the results of this study are presented. The research is discussed in Sect. 5; finally, conclusions are given in Sect. 6.

\section{Measurement Classifications}

\subsection{Aerial photography}

The global positioning system (GPS) was originally developed for military positioning and navigation purposes by the United States of America and was later expanded and employed for civilian positioning measurement. The entire system is composed of 24 satellites that are evenly distributed in six orbital planes. The orbits are almost circular, and the satellites fly at an altitude of approximately $20000 \mathrm{~km}$, orbiting Earth once every $12 \mathrm{~h}$. This ensures that four to seven satellites can be simultaneously observed at any time and from any place worldwide; this is useful for navigation and precise positioning measurement. GPS satellites constantly transmit satellite signals to the ground, whereas ground users employ satellite receivers to receive signals. The distances between satellites and ground receivers, in addition to the baseline vectors between the ground receivers, are obtained using numerous signal characteristics, after which geometry is used to determine the receiver locations, completing navigation positioning and various measurements. A shortcoming of satellite images is their low resolution. 


\subsection{Conventional aerial photography surveying}

Aerial photography refers to taking photographs of the topography of the Earth taken from the air, thus obtaining a bird's-eye view; this is also known as a photomap. The cameras used in aerial photography can be controlled remotely by photographers or can take photographs automatically. The platforms used for aerial photography include airplanes, helicopters, hot air balloons, small airships, rockets, kites, and parachutes. To ensure the stability of aerial photography equipment, advanced equipment such as a SpaceCam is sometimes employed, whereby the incorporated three-axis gyroscope enables the capture of high-quality and stable images even when a lens with a large focal length is used.

\subsection{Ground surveying}

Surveying originated in the construction and land development sectors and is generally referred to as "surveying and mapping" in regions such as China, Taiwan, and Japan. In Hong Kong, the British surveyor industry has continued. Surveyors can be generally classified as building, general practice, land, quantity, planning and development, and property and facility management surveyors. Generally, surveying is an extensively applied technique and not limited to a specific field, although it is unclear in which industry it was first developed; humans have been surveying by one method or another since ancient times. Surveying is a major human approach to the exploration of nature and continues in modern technological society.

\section{Domestic Applications of UAVs}

UAVs have been developed in Taiwan for more than 10 years. The components of a simple UAV generally include unit group GPS receivers, a wireless remote control transceiver, a flight control system, the flight vehicle, and photographic surveillance and sensing equipment. The early development of the technology in Taiwan was mainly oriented toward military application. In recent years, government agencies have been using UAVs in tasks for agriculture, fisheries, animal husbandry, topography, meteorological observation, traffic monitoring, and disaster prevention and control. ${ }^{(11-13)}$ With their all-encompassing applications, UAVs can be expected to play a crucial role in future technology.

\subsection{UAV application in homeland disaster assessment and relief}

UAVs have provided solutions to various problems for engineers. For example, initial feasibility studies and the planning of construction projects conventionally relied on satellite imagery, orthographic images, or photomaps sold by the Aerial Survey Office. After the evaluation and analysis of hard copy data, engineers must conduct on-site investigations and confirmations. This stage is generally difficult and dangerous for worksites that are not easily accessible. In the case of disaster prevention and relief, such as scene after the occurrence of landslide, disaster prevention centers previously determined the appropriate command and 
dispatch on the basis of information obtained through disaster causal analysis, disaster scale and hazard assessment, and follow-up recommendations relayed by frontline staff through phone calls, photos, and videos. Neugirg et al. combined ground control points and UAV images to create orthophotos and 3D point clouds, using the structure from motion software. ${ }^{(14)}$ Aerial photographs of such sites, taken after the weather has stabilized, often reveal the results of devastation, but the golden period during which effective disaster relief can be provided is missed.

\subsection{Flight course planning for UAV surveying}

The difficulties in the postproduction of UAV surveying include those caused by a lowprecision GPS and an inertial navigation system (INS), the use of nonmeasuring cameras, problems in geometric integration between GPS/INS and cameras, a fuzzy image ratio of approximately $1-5 \%$, and the tendency of UAVs to deviate from the flight course because of low wind resistance. These problems can be solved using high overlap rates. In $2 \mathrm{D}$ surveying, the following methods can be taken to enhance accuracy:

(a) A two-way flight that enhances accuracy [Fig. 1(a)];

(b) Forward overlap $\geq 80 \%$ and lateral overlap $\geq 40 \%$ : if thickets are encountered in flat terrain, increasing the overlap rate to forward overlap $\geq 90 \%$ and lateral overlap $\geq 50 \%$ is recommended [Fig. 1(b)];

(c) Two-level (two altitudes) flights increase the overlap range, with one of the flights operated at the corresponding altitude of the configured ground sample distance (GSD) and the other flight operated at an altitude that is 1.5-2 times that of the configured GSD. Forward overlap $\geq 80 \%$. Lateral overlap $\geq 40 \%$ [Fig. 1(c)].

The 3D modeling of flight route planning for aircraft can be roughly divided into the following two types:

(a) Single-building modeling: sufficient overlap rate of $\geq 80 \%$. The camera is tilted at an angle, and the optical axis is not perpendicular to the ground (Fig. 2).

(b) Modeling of an entire area of buildings: sufficient overlap rate of $\geq 80 \%$. The camera is tilted $45^{\circ}$ for shooting (Fig. 3).

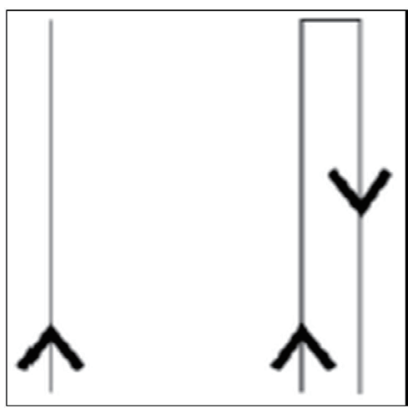

(a)

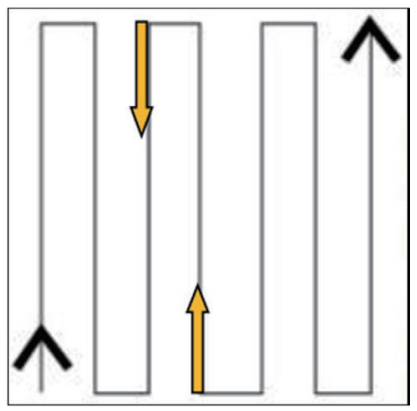

(b)

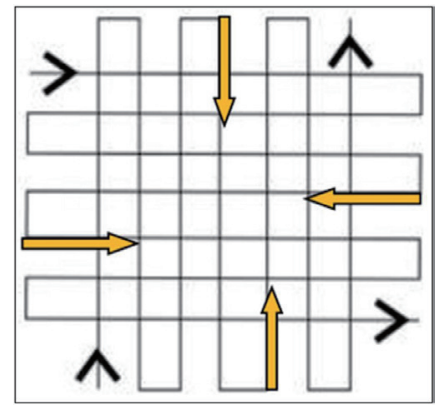

(c)

Fig. 1. (Color online) Flight course to increase surveying accuracy. (a) Two-way flight, (b) overlapping flight, and (c) two-level flight. 


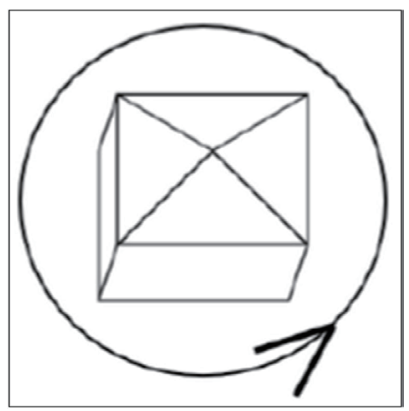

Fig. 2. Single-building flight.

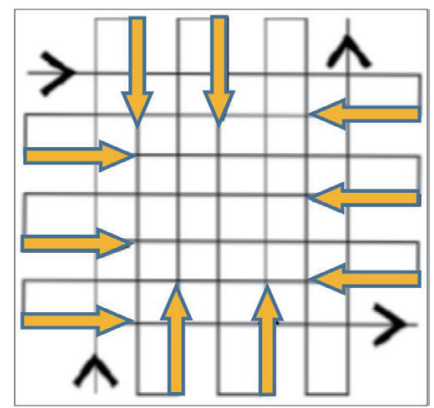

Fig. 3. (Color online) Entire-area-of-buildings flight.

\section{Results}

In this study, an unmanned helicopter equipped with a digital camera was used to take photographs of locations such as hillsides and valleys that are inaccessible to surveyors. Subsequently, 3D models of the hillsides were constructed using the Pix4Dmapper software for use in water and soil conservation planning and design. The following results were obtained.

(a) Selection and operation training of UAV and photography equipment: The UAV used in this study was Phantom 2 Vision+, which was chosen for its suitable performance, low cost, and acceptable availability and maintenance. Despite unmanned remote-controlled helicopters being popular toys, their users must be fully familiar with their operation when given a special task; otherwise, the equipment may be lost if it crashes in an inaccessible area such as a remote stream or valley.

(b)Aerial photographs: The task in this phase was to conduct on-site aerial photography to obtain actual photographs of sites prior to modeling. Figure 4 displays photographs taken at Pingguang Stream in New Taipei City. The flight altitude was $120 \mathrm{~m}$.

(c) Import photos: After the aerial images were obtained into the projector, the system stored the photographs in a database in accordance with their information such as numbering, coordinates, altitude, and flight pattern (Fig. 5). From information such as the coordinates and camera positions of the photographs, the system reconstructed them into a satellite image map for use in subsequent computation. The red dots in Fig. 6 are the coordinates for photographs taken using the UAV, whereas the green lines indicate the flight routes of the UAV.

(d)Computation: Data were computed after the photographic data were imported into the system and databased. The bottom of the screen displayed functions such as initialization, point cloud densification, DSM generation, and orthomosaic. After selecting "Log Output", the screen displayed relevant information regarding the data processing of the system (Fig. 7). Selecting "Processing Options" prompted the system to display the detailed configurations of the "Processing Options" function (Fig. 8). The tasks performed at this stage included the following.

(1) Initialization: this function includes on-site real-time rapid processing and full processing; the latter is further divided into functions such as feature extraction, image matching optimization, and output. 

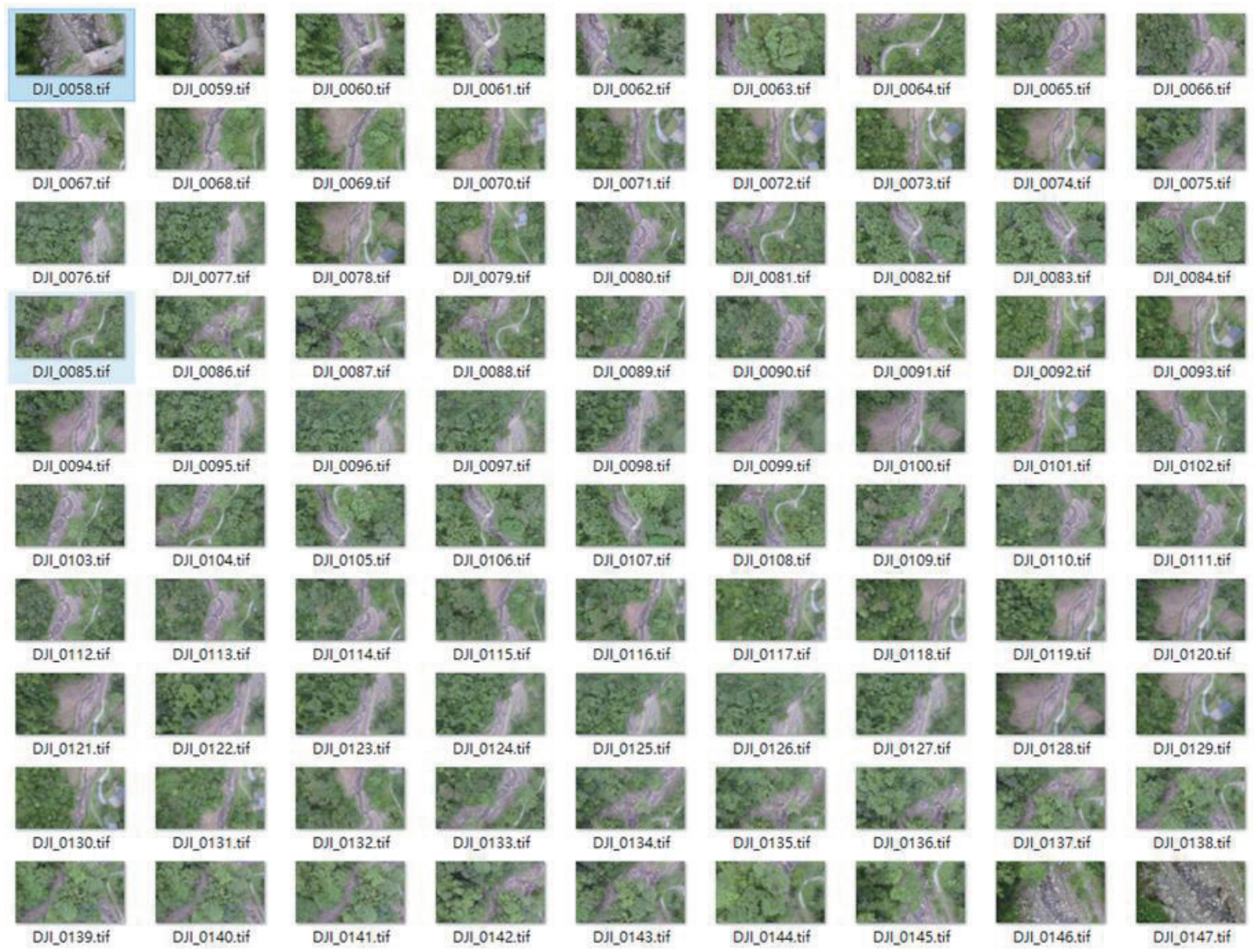

DII_0073.tif

DJl_0074.tif

DJI 0075 .tif
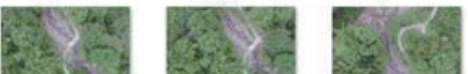

DJI_0081.tif

DII0082.tif

D)_0083.tif

DII_0084.tif
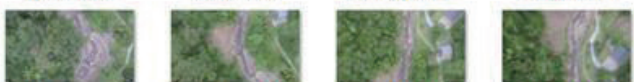

DJI_0090.tif

DII0091.tif

DJI_0092.tif

DJl_0093.tif

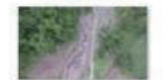

D)__0099.tif
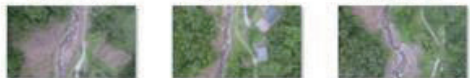

DII_0100.tif

DJl_0101.tif

DII_0102.tif
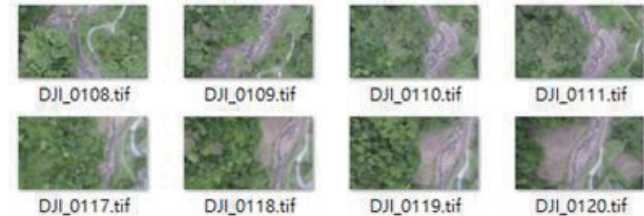

DJ_0111.tif
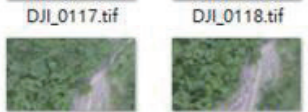

DЛ_0119.tif

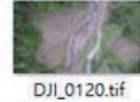

DJl_0126.tif
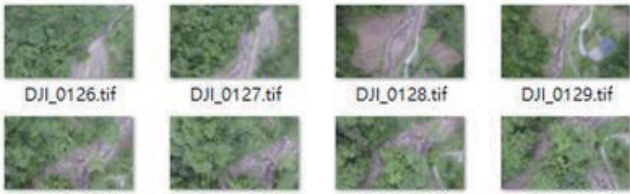

DJ_0128.tif

DII0129.tif

DJI_0135.tif

DII 0136.tif
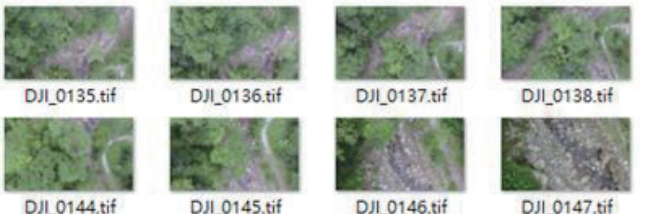

DJI_0138.tif

Fig. 4. (Color online) On-site images taken in this study.

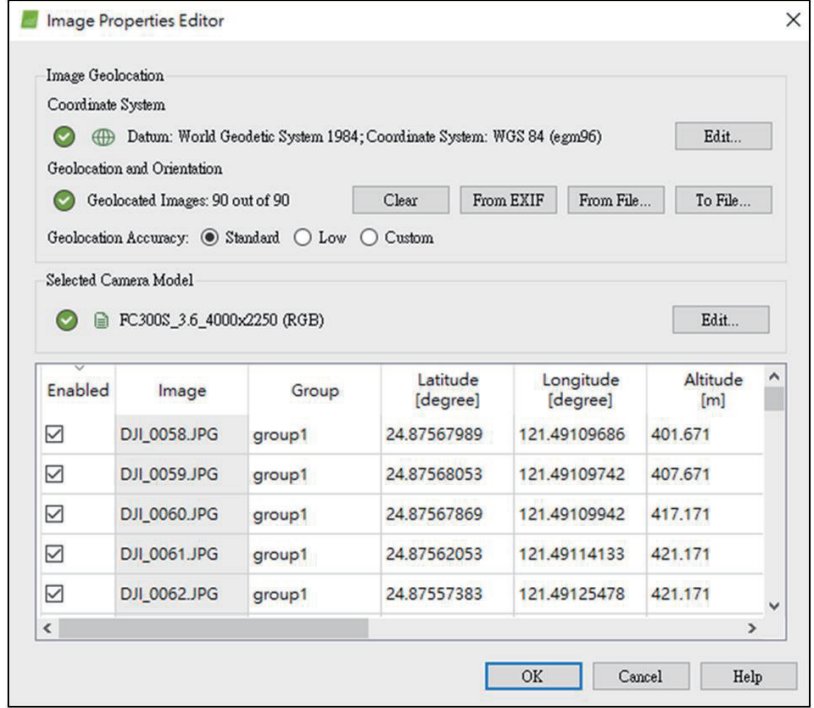

Fig. 5. (Color online) Screenshot of importing the photographs into the system software.

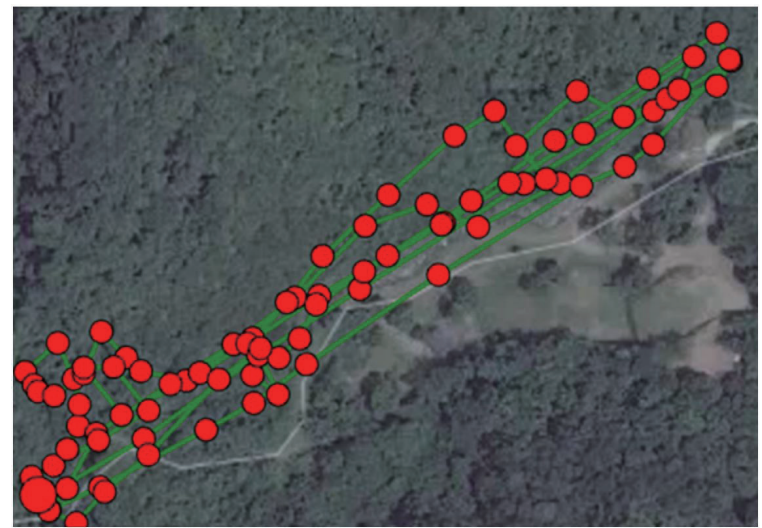

Fig. 6. (Color online) Photograph shooting locations and UAV flight routes. 


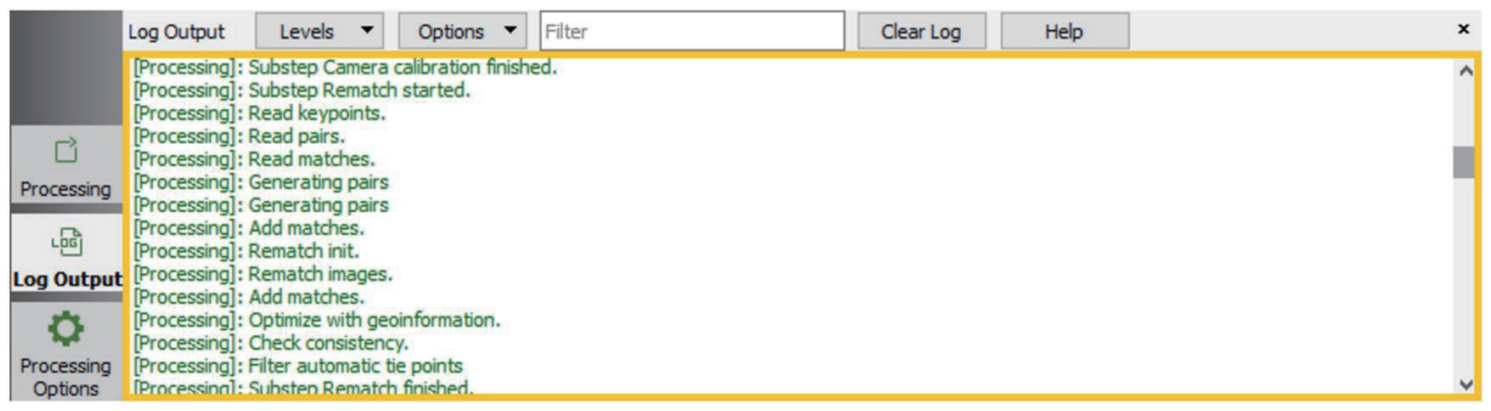

Fig. 7. (Color online) Screenshot of system's data processing.

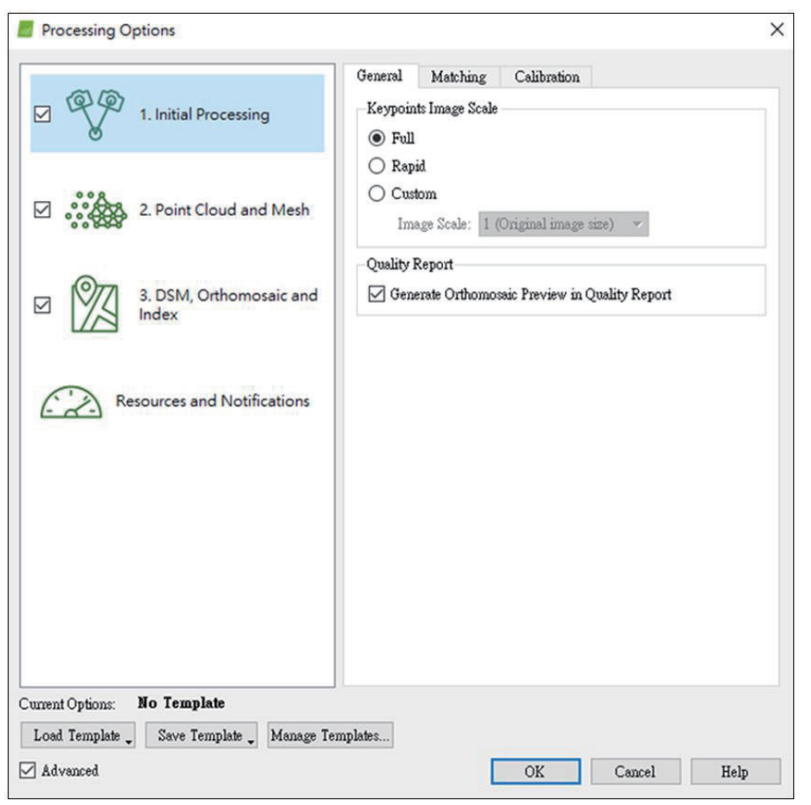

Fig. 8. (Color online) Screenshot of "Processing Options".

(2) Point cloud and mesh model: point cloud densification produces point clouds with filtered noise and a smoothed filter surface in addition to the output of various file formats, such as PLY, XYZ, LAS, and LAZ.

(3) DSM generation and orthomosaic: this function allows the generation and output of files such as raster DSM, grid DSM, and orthomosaic; output file formats include triangular mesh 3D model (.obj), contour lines (.shp, .pdf, .dxf), and 3D pdf.

(e) Luminous cloud editing: at this stage, the system displays the camera locations, flight routes, and reconstructions of camera angles. The left side of the screen shows layers such as cameras, beams, and connection points (Fig. 9). On the basis of the information captured, the system allows users to inspect the number of connection points for each photograph (Fig. 10). In addition, the connections between the initial connection points and the cameras can also be inspected (Fig. 11). Computations of point cloud densification normally consume a vast amount of computer resources and take a long time. The 3D model of the photographed area can be produced from the point cloud once point cloud densification has been completed (Fig. 12); the model can then be applied in the next stage of feasibility assessment and planning design. 


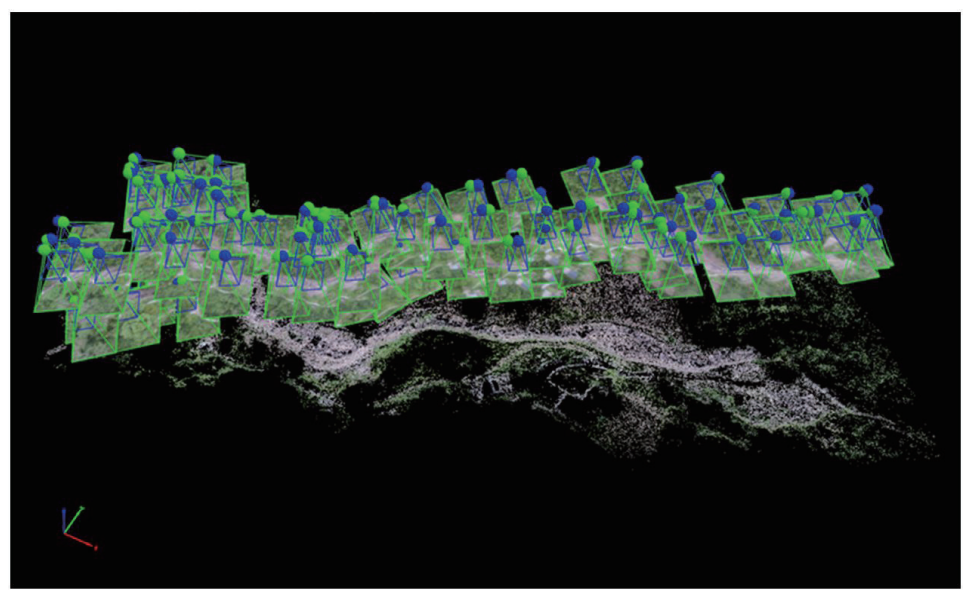

Fig. 9. (Color online) Screenshot of luminous cloud editing.

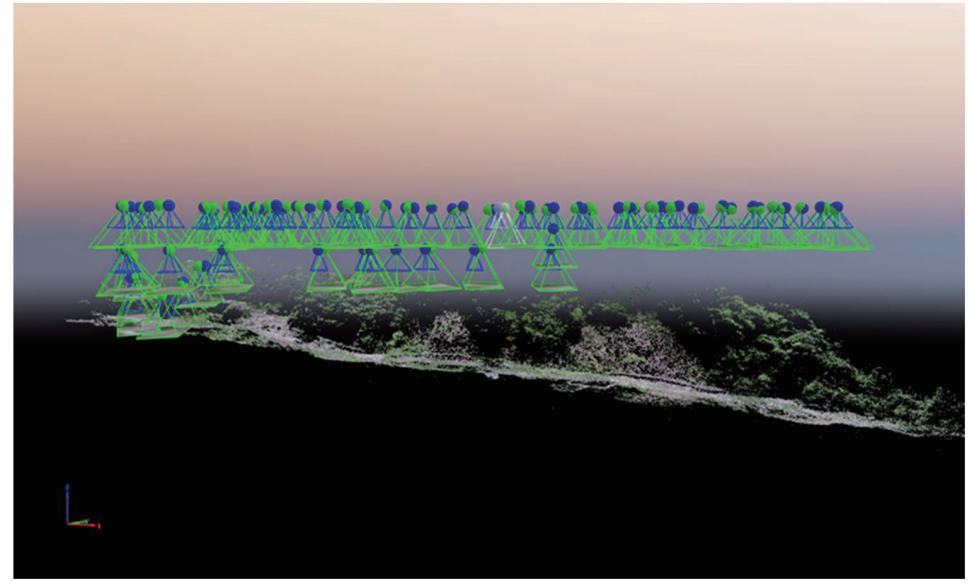

Fig. 10. (Color online) Calculation of camera connection points.

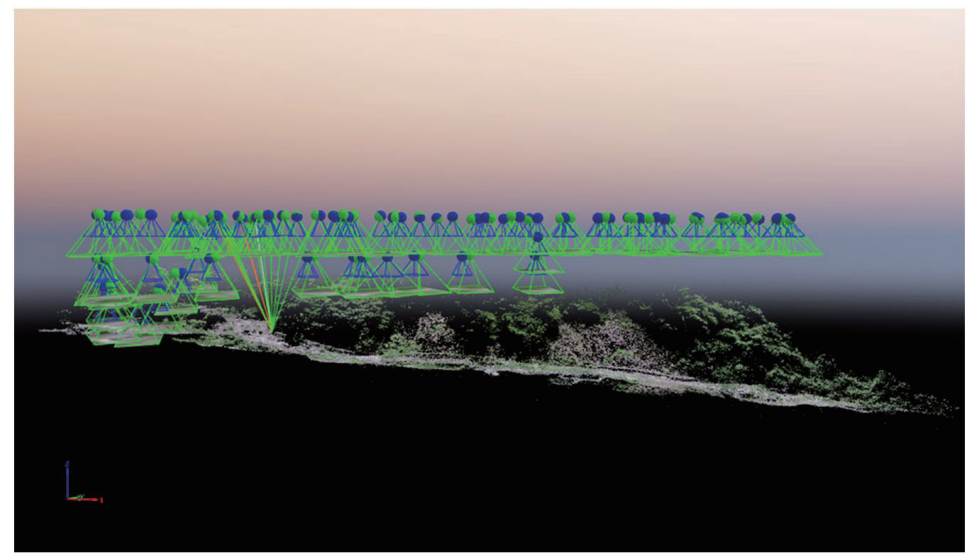

Fig. 11. (Color online) Connections between the connection points and the camera. 

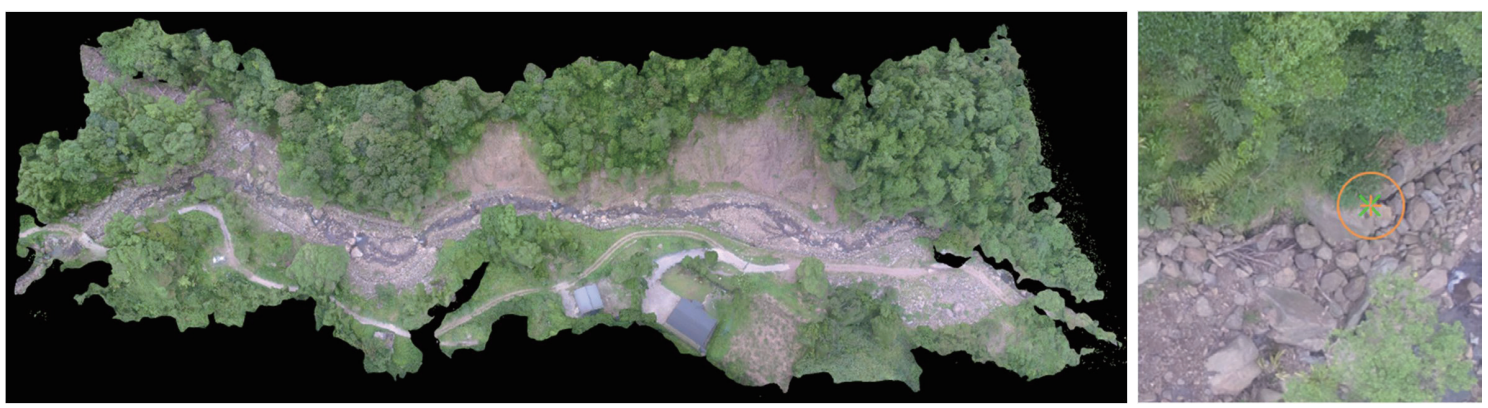

Fig. 12. (Color online) 3D model obtained after point cloud densification.

\section{Discussion}

In this study, the UAV was flown above the target area to photograph orthoimages. The images were imported into the Pix4Dmapper software to obtain point cloud files of the target area and reconstruct a visualized DSM. This can effectively assist the planning and design departments to construct 3D models of hillsides that closely resemble the actual site of a water and soil conservation project.

In a future study, the 3D GIS can be combined with the BIM system to perform viewing angle adjustment, attribute query, profile presentation, transparent view, simple material quantity statistics, and other functions.

\section{Conclusion}

In this study, UAV applications, remote sensing technology, and aerial images were used. We demonstrated the rapid construction of 3D models of hillsides employing an unmanned helicopter, a camera, and Pix4Dmapper. Taking the project described in this study as an example, one engineer, $2 \mathrm{~h}$ of aerial photography, $4 \mathrm{~h}$ of data computation, and $1 \mathrm{~h}$ of image output were required; four hectares of terrain was surveyed within a total of 7 h. If this surveying had been conducted using conventional approaches, it would have taken approximately three engineers and 3 working days (a total of 9 manpower days). Thus, approximately $90 \%$ of the workforce and work time could be saved by using the method proposed in this study. The contributions of the present study are as follows.

(a) After obtaining photographs of hillsides by UAV aerial photography, a 3D model of the hillsides can be rapidly constructed using Pix4Dmapper. This method can be used for planning and design purposes by engineering consultants and other units, eliminating the need for long and difficult travel to complete a survey. The task of 3D modeling can be performed through software computation.

(b) The method proposed in this study will assist on-site surveying and mapping personnel to effectively collect construction site information, solving inaccessibility problems due to difficult terrain and thereby avoiding potential accidents involving staff members surveying and mapping dangerous areas. 
(c) The convenience of unmanned helicopter surveying enables the collection of photographs and videos on-site at different altitudes and angles. This increases the accuracy of surveying and mapping and decreases the number of planning and design imperfections due to inadequate information and data errors, which results in the waste of resources and potential loss of life and property.

(d) 3D models of hillside areas can be rapidly and automatically constructed from aerial photographs using the Pix4Dmapper software, effectively saving manpower and time so that critical hillside planning and design can be completed more quickly and on schedule. In addition to its potential use for public infrastructure, the proposed method is also be effective in the prevention of disasters caused by heavy rain such as those occurring during typhoon season in the extensive rainy mountain areas of Taiwan, which are also subject to frequent earthquakes.

\section{References}

1 Y.-C. Shiau, Y.-P. Chiu, H.-C. Liu, and J.-C. Chan: ICIC Express Lett. Part B 5 (2014) 205.

2 P.-H. Hsu: Sens. Mater. 28 (2016) 455.

3 H. Zhang, Z. Song, J. Yang, Q. Yang, C. Wang, and R. Li: Trans. Chin. Soc. Agric. Mach. 48 (2017) 112.

4 K. T. Chang, G. W. Lee, L. S. Liang, J. K. Liu, and F. C. Yu: 2017 IEEE Int. Geoscience and Remote Sensing Symposium (IGARSS) (2017) 6275.

5 E. C. Barrett: Introduction to Environmental Remote Sensing (Routledge, New York, 2013).

6 T. Lillesand, R. W. Kiefer, and J. Chipman: Remote Sensing and Image Interpretation (John Wiley \& Sons, NJ, USA, 2014).

7 K.-T. Chang and W.-L. Hsu: 2018 IEEE Int. Conf. Applied System Invention (ICASI) (2018) 168.

8 S. Chen, H. Yang, S. Wang, and Q. Hu: Sustainability 10 (2018) 3138.

9 F. Rossi, A. Fritz, and G. Becker: Sustainability 10 (2018) 2227.

10 L. Wallace, A. Lucieer, C. Watson, and D. Turner: Remote Sens. 4 (2012) 1519. https://www.mdpi.com/20724292/4/6/1519

11 H. Wenting, L. Guang, and Y. Mengchan: Trans. Chin. Soc. Agric. Mach. 48 (2017) 140.

12 H. Wenting, G. Congcong, and Z. Liyuan: Trans. Chin. Soc. Agric. Mach. 47 (2016) 270.

13 C. Y. Hsiao, H. P. An, C. K. Chen, J. Y. Rau, and S. C. Chen: J. Chin. Inst. Civ. Hydraul. Eng. 27 (2018) 213.

14 F. Neugirg, M. Stark, A. Kaiser, M. Vlacilova, M. Della Seta, F. Vergari, J. Schmidt, M. Becht, and F. Haas: Geomorphology 269 (2016) 8.

\section{About the Authors}

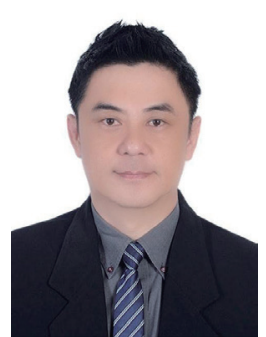

Wei-Ling Hsu received his Ph.D. degree in 2014 from the Department of Civil Engineering and his Master's degree in architecture in 2009 from Chung Hua University in Taiwan. From 2009 to 2017, he served as an adjunct assistant professor at Chung Hua University and Minghsin University of Science and Technology. He is currently working at the Huaian Key Laboratory of Geographic Information Technology and Applications, School of Urban and Environmental Science, Huaiyin Normal University, China. His research expertise includes the application of satellites and unmanned aerial vehicles in ground-level environmental monitoring, urban traffic, urban and rural planning, and the incorporation of data analysis in fuzzy multiplecriteria decision making for cities and environments. 


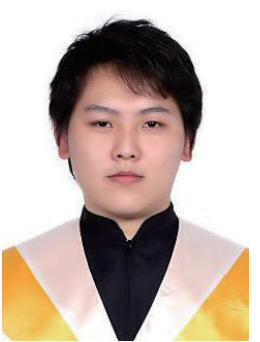

Ji-Yun Jhuang received his B.S. degree from Ta Hwa University of Science and Technology, Taiwan, in 2007. He received his M.S. degree from Hsuan Chuang University, Taiwan, in 2010. From 2010 to 2016, he was a Ph.D. student and R.A. in Chung Hua University. He is currently a reserved director in Cathay Life Insurance Company, Ltd., Taiwan. His research interests are in IoT and smart homes.

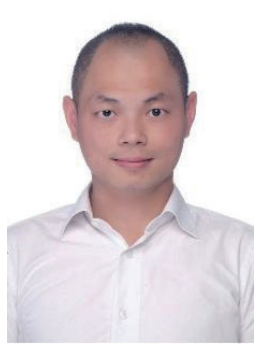

Yi-Sian Chen received his B.S. degree from Huafan University, Taiwan, in 2007. He received his M.S. degree from Chung Hua University, Taiwan, in 2012. Since 2012, he has been a graduate student in a Ph.D. program at Chung Hua University, Taiwan. His research interests are in historical building research, public art spatial decision-making and analysis, and senior housing decision-making and analysis.

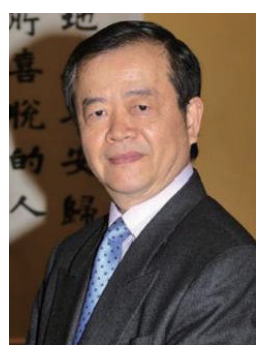

Yan-Chyuan Shiau received his B.S. degree from National Taiwan Institute of Technology, Taiwan, in 1980 and his M.S. and Ph.D. degrees from Texas A\&M University, U.S.A., in 1988 and 1992, respectively. From 2000 to 2012, he was an associate professor at Chung Hua University, Taiwan. Since 2013, he has been a professor at Chung Hua University, Taiwan. His research interests are in ICT, IoT, and smart homes.

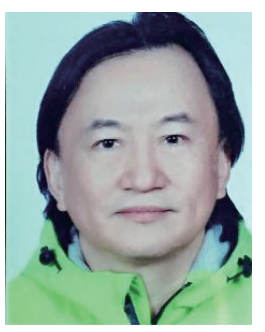

Tian-Yow Chern received his B.S., M.S., and Ph.D. degrees from Kassel University, Germany, in 1990, 1992, and 1995, respectively. Since 1998, he has been an associate professor at Chung Hua University, Taiwan. His research interests are in architectural theory, space aesthetics, and community building. 